Rwanda to participate in two "opioids in palliative care" workshops, targeting pharmacists and medical leads in an attempt to demystify opioid use, reduce opiophobia and highlight the benefits of collaborative, multidisciplinary teamwork.

We were asked to deliver several sessions including overview of palliative care in the world; opioid fears/myths, diversion, addiction; pain management, clinical cases and more! With six weeks to prepare, we spent evenings and weekends brainstorming innovative ways to engage our African colleagues from simple games and physical props, to quizzes and small group work, one patient even recorded a video about morphine and how it helped them live despite a palliative diagnosis.

The workshops were well received and delegates entered into the spirit of and appeared to thrive on our interactive exercises. Analysis of learning outcomes as established using pre and post course assessments showed significant improvement in opinions and understanding and ongoing communication with our Rwandan colleagues has shown morphine use in pain management continues to increase. We will also return to Rwanda later this year as part of a second THET project.

Whilst large formulated projects are vital in promoting good palliative care around the world the role of individual health professionals and the forming of personal links should be integral to this, so many of us have skills to share and could contribute and we hope that by sharing our experience we might encourage others to do the same as we are proof that from chance beginnings positive change can occur.

\section{P-231 DEVELOPING END OF LIFE CARE LEARNING OUTCOMES FOR THE WORKFORCE ACROSS YORKSHIRE AND THE HUMBER: A STRATEGIC APPROACH}

${ }^{1}$ Vanessa Taylor, ${ }^{2}$ Grace Jeffrey, ${ }^{2}$ June Toovey. ${ }^{1}$ University of York, York, UK; ${ }^{2}$ Health Education Yorkshire and the Humber

\subsection{6/bmjspcare-2016-001245.252}

In 2012, End of Life Care (EoLC) Leaning Outcomes (LOs) were proposed by an expert group, part of Yorkshire and Humber Strategic Health Authority EoLC work stream. The continuing development of the EoLC LOs has been supported by Health Education Yorkshire and Humber.

The EoLC LOs have been produced in the context of ongoing national developments all advocating that, for improvements to be achieved in the delivery of palliative and EoLC, workforce development is required. This is the first framework which identifies outcomes for the workforce involved in palliative and EoLC in any setting. The EoLC LOs are intended for use as part of education provision and workforce development to support improvements in the delivery of high quality EoLC delivered by:

1. Unregistered support workers in health and social care

2. Pre-qualifying health and social work students

3. Registrants providing palliative care approach/general palliative care

4. Registrants providing specialist palliative care.

As part of this initiative, there have been two pilots of their use in clinical practice, mapping exercises by higher education institutions (HEIs) against pre-qualifying and CPD programmes, and an expert group workshop to scrutinise the LOs. Feedback from these pilots will be presented. Feedback confirms that the EoLC LOs have the potential to deliver benefits for the workforce, employers, commissioners, patients and the public including:

- Developing personal career goals

- Developing job/role descriptions

- Assessing clinical knowledge and skills at different levels of EoLC practice

- Performance appraisal

- Enabling professional revalidation

- Enabling organisations and commissioners to respond to national policy related to local implementation and delivery of EoLC.

In press publications for this initiative:-

1. End of Life Care Learning Outcomes

2. Workplace Development Record

3. Mapping Tool for education providers

4. Sub-sets for 'One Chance to Get it Right' and for mandatory/ priority EoLC education and training

5. Mapping to Skills for Health Competences.

\section{P-232 LOTHIAN CARE ASSISTANT DEVELOPMENT PROGRAMME - A SOCIAL CARE EDUCATION PROJECT}

Lyndsay Cassidy. Marie Curie, Edinburgh, UK

10.1136/bmjspcare-2016-001245.253

Background and aims With Scottish Government's integration of health and social care, social care teams are increasingly leading the care for elderly clients and their families who are living with a terminal illness and also have multiple complex care needs. Addressing the learning needs of social care workers is essential to ensure the provision of high quality palliative care for families' in their preferred place of care, and to reduce emergency admissions to hospital where avoidable.

The education programme increases the knowledge and confidence of social care workers in a care home or home care setting in Lothian, in caring for people and their families living with a terminal illness. It increases the ability to identify people with palliative care needs and improves awareness of community services available to support patients as they approach end of life.

Methods Through consultation, a tiered approach to learning was developed. Care workers attended a single training day, with a portion going on to complete an online module. The module was contextualised to reflect the Lothian focus. Care workers were supported in their care setting by a workplace mentor. Mentorship training and support was provided by the programme team.

Results 500 social care workers will have completed the single day training and 75 will have gone on to have completed the online professional development module by the conference date. Single day evaluation, pre- and post-knowledge and confidence questionnaire and focus groups are being used to evaluate the programme and its transferability.

Conclusions To achieve the aims set out in the Strategic Framework for Action on Palliative and End of Life Care, it is essential that social care teams are adequately supported and trained to provide high quality palliative care for people and their families living with a terminal illness. 